\title{
Serum estradiol level on the day of trigger as a predictor of number of metaphase II oocytes from IVF antagonist cycles and subsequent impact on pregnancy rates
}

Héctor Salvador Godoy Morales ${ }^{1}$, Miguel Loyo Guiot ${ }^{1}$, Germán Gabriel Palacios López ${ }^{1}$, Daniel Vieyra Córtes ${ }^{1}$, Berenice Flores Maldonado ${ }^{1}$, Hilda Sánchez Hernández ${ }^{1}$, Griselda Claribel Reyes Torres ${ }^{1}$, Francisco Miguel Rojas Camacho $^{1}$, Gabriela Ayala Montoya ${ }^{1}$

${ }^{1}$ Angeles Pedregal Hospital, Assisted Reproduction Unit, Mexico City, Mexico

\begin{abstract}
Objective: This study aimed to examine the association between serum estradiol levels and number of metaphase II oocytes harvested after in vitro fertilization cycles used in embryo transfers and the subsequent impact on pregnancy rates.

Methods: This observational analytical retrospective study was carried out in 2010-2018 at the Angeles del Pedregal Hospital. It included 181 cases and looked into the number of metaphase II oocytes to predict pregnancy rates. Statistical analysis was based on the calculation of correlations between variables and logistic regressions.

Results: Estradiol levels increased with the number of oocytes by a median correlation $(r=0.482, p=0.000)$. On the day of trigger, estradiol levels predicted the number of retrieved oocytes with $23 \%$ reliability $(R 2=0.232$, $p=0.000)$; a linear trend correlation of $r=0.489, p=0.000$ was found between estradiol levels on the day of trigger and number of metaphase II oocytes.

Conclusions: Serum estradiol on the day of trigger as a predictor of metaphase II oocytes in antagonist cycles encourages greater oocyte maturity and fertilization, whereas, in isolation, it does not determine the pregnancy achievement.
\end{abstract}

Keywords: estradiol, oocyte retrieval, trigger day, antagonists, pregnancy rate

\section{INTRODUCTION}

The main objective of controlled ovarian stimulation (COS) in in vitro fertilization (IVF) cycles is to produce a large number of mature oocytes. On the other hand, elevated secretion of ovarian steroid hormones is strongly associated with COS, and in this regard, serum estradiol $\left(E_{2}\right)$ levels may increase more than 10 times compared to levels found during spontaneous cycles. Serum estradiol levels have been used as a predictor at the time of oocyte retrieval (Macklon et al., 2006; Pittaway \& Wentz, 1983).

Estradiol is the most potent estrogen ( $17 \beta$-estradiol). It is synthesized by ovarian granulosa cells from androstenedione and testosterone, as the main product of ovary endocrine secretion (Wu et al., 2007; Brown, 1978). In the natural ovulatory cycle, estradiol concentrations range from 200 to $400 \mathrm{pg} / \mathrm{mL}$ just before luteinizing hormone (LH) levels peak. In exogenously stimulated cycles, similar concentrations are expected per follicle. However, the levels reported in the literature are very dissimilar (Speroff \& Fritz, 2007). Additional studies based on cycles with antagonist protocols should be performed to establish more reliable parameters and reflect more accurately their effects in the Mexican population.

\section{MATERIALS AND METHODS}

\section{Patient Characteristics}

This observational descriptive cross-sectional retrospective study included the records of patients seen at the Fertility Clinic of the Angeles del Pedregal Hospital in Mexico City, from 2010 to 2018. The study included patients submitted to IVF diagnosed with infertility of any kind (primary or secondary) aged 18-56 years. The antagonist protocol was started when the patients reached a follicle size of $14 \mathrm{~mm}$. Additional information such as serum estradiol levels prior to the trigger and cycle outcome, number of retrieved oocytes, number of metaphase II (MII) oocytes, and pregnancy rate were also captured. Patients achieving pregnancy with donor eggs or with associated male factor infertility and patients with exogenous estradiol supplementation were excluded. The social and clinical characteristics of the study group are shown in Table 1.

\section{Stimulation Protocol}

Since infertility is a multifactorial condition, this study considered the most common diseases - including polycystic ovary syndrome (PCOS), fibroids, polyps and hypothyroidism - to decrease information bias. Patients started stimulation on day 2 with recombinant gonadotropin in most cases or an antagonist protocol with Cetrotide 0.25 $\mathrm{mg}$, (when the follicle reached $14 \mathrm{~mm}$ ), although some used highly purified urinary gonadotropin (Gonal F 150300 IU/Merional 225-300 IU) in their cycles. Doses were established based on patient baseline characteristics (age, weight, $\mathrm{FSH}$, anti-Müllerian hormone and antral follicle count) and were adjusted from the sixth day of stimulation based on ultrasound controls. Choriomon 10,000 IU was used in final oocyte maturation in all cases.

\section{In Vitro Fertilization and Embryo Transfer}

Oocyte maturation was performed 36 hours prior to follicular puncture using urinary HCG; in patients at risk of ovarian hyperstimulation, a GnRH analogue was used at a dosage of $0.2 \mathrm{mg}$. In all cases, the Lübeck or antagonist protocol was used to prevent spontaneous ovulation. $\mathrm{GnRH}$ antagonists (Cetrorelix) were used at a dosage of $0.25 \mathrm{mg}$ when a follicle greater than $14 \mathrm{~mm}$ was observed.

\section{Hormonal Determination}

Serum estradiol measurements were performed in a single laboratory, belonging to the Angeles del Pedregal Hospital, since the samples were taken during the last appointment and sometimes on the day of retrieval. It is important to mention that the same medical team performed oocyte retrievals and estradiol level measurements.

\section{Statistical Analysis}

Patients were selected based on non-probability sampling and the study method was non-participant 
Table 1. Clinical characteristics of the study group

\begin{tabular}{|c|c|c|c|}
\hline & Median & Minimum & Maximum \\
\hline Age (years) & 35.9 & 18 & 56 \\
\hline Body mass index $\left(\mathrm{kg} / \mathrm{m}^{2}\right)$ & 18.5 & 36 & 24.5 \\
\hline Height (m) & 1.62 & 1.57 & 1.77 \\
\hline Baseline E2 (pg/ml) & 54.15 & 5 & 278 \\
\hline Baseline anti-Müllerian hormone $(\mathrm{ng} / \mathrm{ml})$ & $1.95 \pm 2.08$ & 0.003 & 8.11 \\
\hline Baseline FSH (UI/mI) & 8.56 & 0.20 & 109 \\
\hline Estradiol average level on the day of trigger per metaphase II oocyte (pg/ml) & 381 & 293.2 & 468.8 \\
\hline $\begin{array}{l}\text { Estradiol by age group }(\mathrm{pg} / \mathrm{ml}) \\
<35 \text { years } \\
36-39 \text { years } \\
>40 \text { years }\end{array}$ & $\begin{array}{c}304.01 \\
349 \\
273\end{array}$ & $\begin{array}{c}41.36 \\
54.5 \\
40.16\end{array}$ & $\begin{array}{l}1086 \\
1118 \\
1375\end{array}$ \\
\hline
\end{tabular}

observation. Data was collected from 181 records of female patients with infertility aged $18-56$ years seen at the Fertility Clinic of the Angeles del Pedregal Hospital in Mexico City from 2010 to 2018 and recorded in data collection cards. No patients were lost or excluded from the study, since it included everyone whose records contained the measurements analyzed in the study.

Patients were divided into three age-based groups: Group I: patients aged $\leq 35$ years; Group II: patients aged 36 and < 40 years; Group III: patients aged $\geq 40$ years. The data sets analyzed in the study were captured from patient medical records and recorded in data collection cards.

The data from the medical records were reviewed and submitted to statistical analyses (Microsoft Excel ${ }^{\odot}$ and IBM SPSS Statistics ${ }^{\odot}$, Version 20). Inferential statistics was used to derive central tendency and dispersion measures for quantitative variables and proportions for qualitative variables. Pearson's correlation coefficient was used to analyze the correlations between quantitative variables following a normal distribution. Spearman's rank correlation coefficient was used to analyze variables not following a normal distribution, as well as the mean comparison tests for ANOVA independent variables of a pathway for normal distribution quantitative variables, and the determination coefficient obtained by logistic regression for predictor variables.

The Research Committee of the Angeles del Pedregal Hospital approved this study, which strictly adhered to the current guidelines of the General Health Law, Chapter I of the Ethical Aspects of Human Beings Research, Article 17.

\section{RESULTS}

The medical records of 181 patients meeting the following inclusion criteria were analyzed: mean age of $35.9 \pm 6.19$ years woman (18-56); baseline $\mathrm{E}_{2}$ of $54.15 \pm$ $53.63 \mathrm{pg} / \mathrm{ml}$ (5-278); baseline anti-Müllerian hormone of $1.95 \pm 2.08 \mathrm{ng} / \mathrm{ml}(.003-8.11)$; baseline FSH of $8.56 \pm 12.76$ $\mathrm{IU} / \mathrm{ml}$ (0.20-109). The average estradiol level on the day of trigger was $217.8 \pm 217.8 \mathrm{pg} / \mathrm{ml}$ per retrieved oocyte and $381 \pm 87.8 \mathrm{pg} / \mathrm{ml}$ per metaphase II oocyte.

In terms of patient age, the following mean $E_{2}$ levels were observed in each age group: $304.01 \pm 208.1 \mathrm{pg} / \mathrm{ml}$ $(41.36-1086)$ in patients aged $\leq 35$ years; $349 \pm 217.01$ $\mathrm{pg} / \mathrm{ml}(54.5-1118)$ in patients aged 36 to 39 years; and $273 \pm 221.75 \mathrm{pg} / \mathrm{ml}(40.16-1375)$ in patients aged 40 and over. Mean $\mathrm{E}_{2}$ levels were statistically different between groups (ANOVA 3.94, gl 2, $p=0.021$ ).
The correlation between serum estradiol levels and number of retrieved oocytes and MII oocytes was assessed. In general terms, the analysis of $E_{2}$ levels and number of retrieved oocytes found that when E2 levels increased, the number of retrieved oocytes also increased. This correlation is of average strength and significant $(r=0.482$, $p=0.000$ ), particularly in the study groups in which the number of oocytes retrieved depended on $E_{2}$ levels at a constant ratio. We found a positive correlation between number of oocytes and estradiol levels in the same age group, meaning that higher levels of estradiol and larger numbers of retrieved oocytes $(r=0.482, p=0.000)$ presented a correlation of average strength. Correlations are deemed strong when $r>.75$.

Logistic regression analysis found a goodness-of-fit between $E_{2}$ levels on the day of trigger and number of retrieved oocytes of $23 \%(R 2=0.232, p=0.000)$, as shown in Figure 1.

Correlation analysis identified an association of average strength $(r=0.489, p=0.000)$ between $E_{2}$ levels on the day of trigger and number of MII oocytes. Logistic regression analysis found a goodness-of-fit of $23 \%\left(R^{2}=0.239\right)$, as shown in Figure 2.

\section{Assessment of Estradiol Levels by Groups}

After analyzing the general findings discussed above, we decided to analyze three different groups based on their respective serum $E_{2}$ levels, as follows: Group 1 with levels up to $1000 \mathrm{pg} / \mathrm{ml}$; Group 2: $\mathrm{E}_{2}$ levels from 1001 $\mathrm{pg} / \mathrm{ml}$ to $3000 \mathrm{pg} / \mathrm{ml}$; and Group 3 with levels >3001 pg/ $\mathrm{ml}$. The mean ages of the patients in each of the groups $(36.7,35$, and 37.05 years) were not statistically different $(p=0.106)$, as also seen in baseline $\mathrm{E}_{2}(p=0.358)$ and $\mathrm{FSH}$ $(p=0.362)$ levels and number of follicles by the end of the stimulation $(p=0.179)$.

Nevertheless, there were noticeable differences $(p=0.000)$ in the number of retrieved oocytes in Group 3 , in which the mean number of retrieved oocytes was $16.51 \pm 8.28$ and MII oocytes was $14.16 \pm 7.49$, thus confirming again that the higher the $\mathrm{E}_{2}$ levels the greater the number of oocytes obtained, with impacts seen also on higher rates of oocyte maturity and fertilization $(p=0.000)$, as shown in Table 2.

\section{Group}

Association between Estradiol and Pregnancy by

Regarding the association between $\mathrm{E}_{2}$ and pregnancy, the evaluation of $E_{2}$ levels on the day of trigger did not reveal statistically significant results $(p=0.681)$, which 


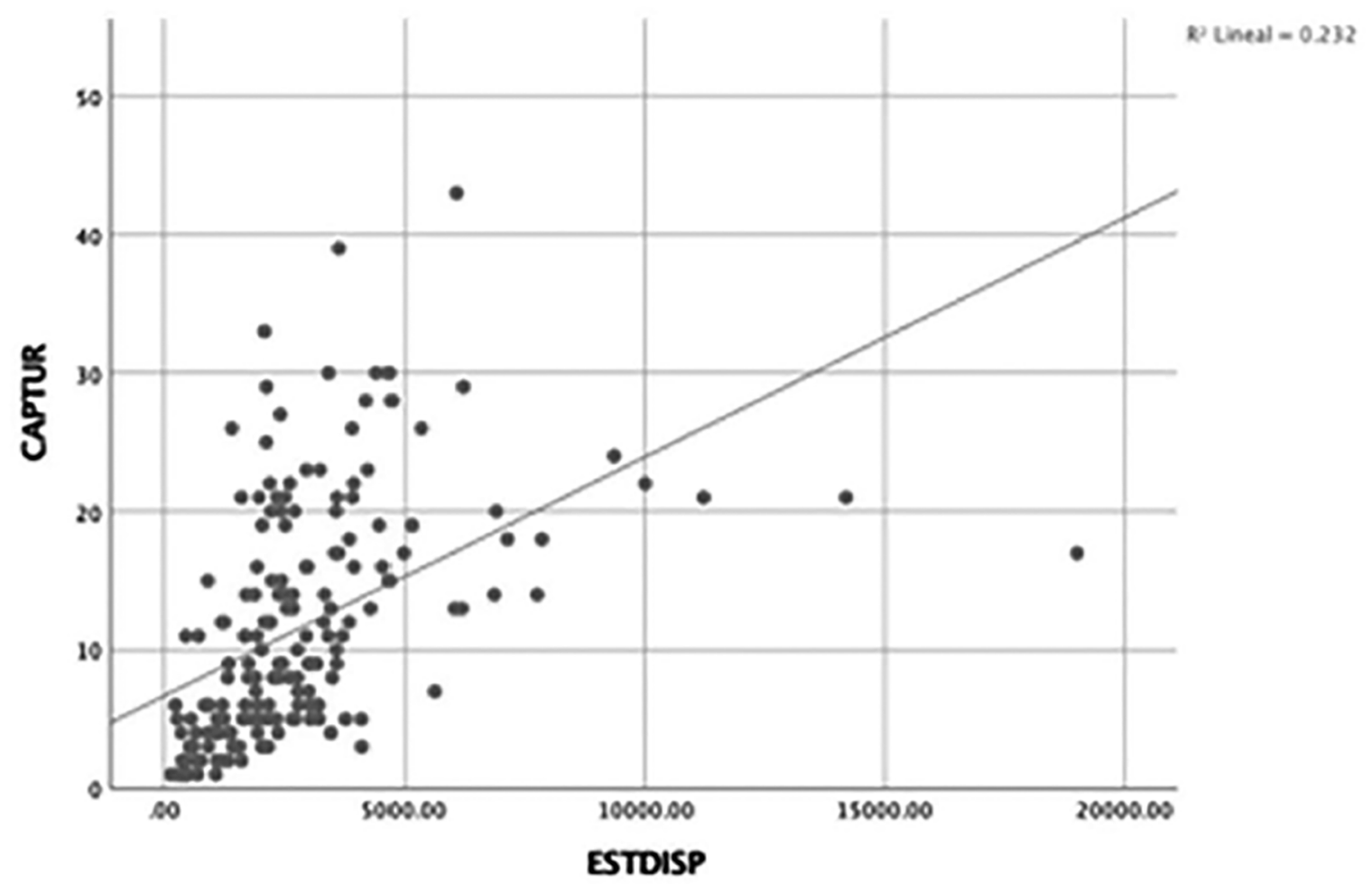

Analysis of variance (retrieved oocytes)

Source (model)

Regression

Residual

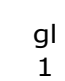

178

Coefficients (retrieved oocytes)

Model term

Constant

Estradiol levels on trigger day

$\begin{array}{cc}\text { Estimation } & \text { Error tip } \\ 6.668 & 0.862 \\ .002 & 0.000\end{array}$

Sum $^{2}$
3008.78

9950.21

Figure 1. Analysis between the number of retrieved oocytes and E2 levels on the day of trigger. means that $E_{2}$ levels were not associated with achieving pregnancy. This was further corroborated as parents were sorted into groups in terms of $\mathrm{E}_{2}$ levels as in the previous section: In group 1 , patients with $\mathrm{E}_{2}$ levels up to $1000 \mathrm{pg} /$ $\mathrm{ml}$ had a pregnancy rate of $32 \%$; in group 2 with $\mathrm{E}_{2}$ levels from $1001 \mathrm{pg} / \mathrm{ml}$ to $3000 \mathrm{pg} / \mathrm{ml}$, the pregnancy rate was $16 \%$; and in group 3 with $\mathrm{E}_{2}$ levels > $3001 \mathrm{pg} / \mathrm{ml}$, the pregnancy rate was $22.6 \%$. Comparisons between groups for pregnancy, miscarriage, and live birth rates did not find statistically significant differences $(p>0.05)$, as shown in Table 3. Therefore, serum $E_{2}$ levels alone are not useful in estimating which patients will achieve pregnancy.

\section{DISCUSSION}

Controlled ovarian stimulation (COS) is a crucial step in current assisted reproductive technologies to obtain mature follicular cohorts. This is inevitably associated with supraphysiological levels of estradiol. Measuring serum $E_{2}$ levels is part of the basic protocol in almost every assisted reproduction center in the world; in addition to predicting the number of expected oocytes, it is also useful in preventing inherent complications from stimulation cycles.

Establishing a more specific serum $\mathrm{E}_{2}$ value as a prognostic marker at the time of controlled ovarian stimulation provides for a more accurate picture of the expected outcomes.
The results seen in this study showed that $E_{2}$ levels on the day of trigger predicted the number of retrieved oocytes with $23 \%$ reliability $\left(R^{2}=0.232, p=0.000\right)$, and that the number of oocytes to be retrieved on average is linked to a value of $300 \mathrm{pg} / \mathrm{ml}$ in antagonist protocol cycles, unlike classic studies, which reported very wide ranges of estradiol levels (200-400 pg/ml per oocyte) (Speroff \& Fritz, 2007).

After consulting the available global literature, no reports were found correlating such accurate pre-trigger estradiol levels and number of retrieved oocytes and MII oocytes obtained from ovarian stimulation cycles using antagonists (Agard et al., 2007).

Secondary analysis of the estradiol levels of patients who achieved pregnancy after ovarian stimulation cycles found no significant difference or different values when comparing their results against the patients who did not become pregnant. In group 1 , the pregnancy rate was $32 \%$; in group 2 it was $34.8 \%$; and in group 3 it was $32.8 \%$. The pregnancy, miscarriage, and live birth rates of the groups were not statistically different $(p>0.05)$. The latter findings agree with a study in which serum estradiol, progesterone and B-HCG levels were analyzed; the authors found that elevated estradiol levels were not statistically associated with achieving pregnancy (Carmona et al., 2003); another study, however, reported that high $\mathrm{E}_{2}$ 


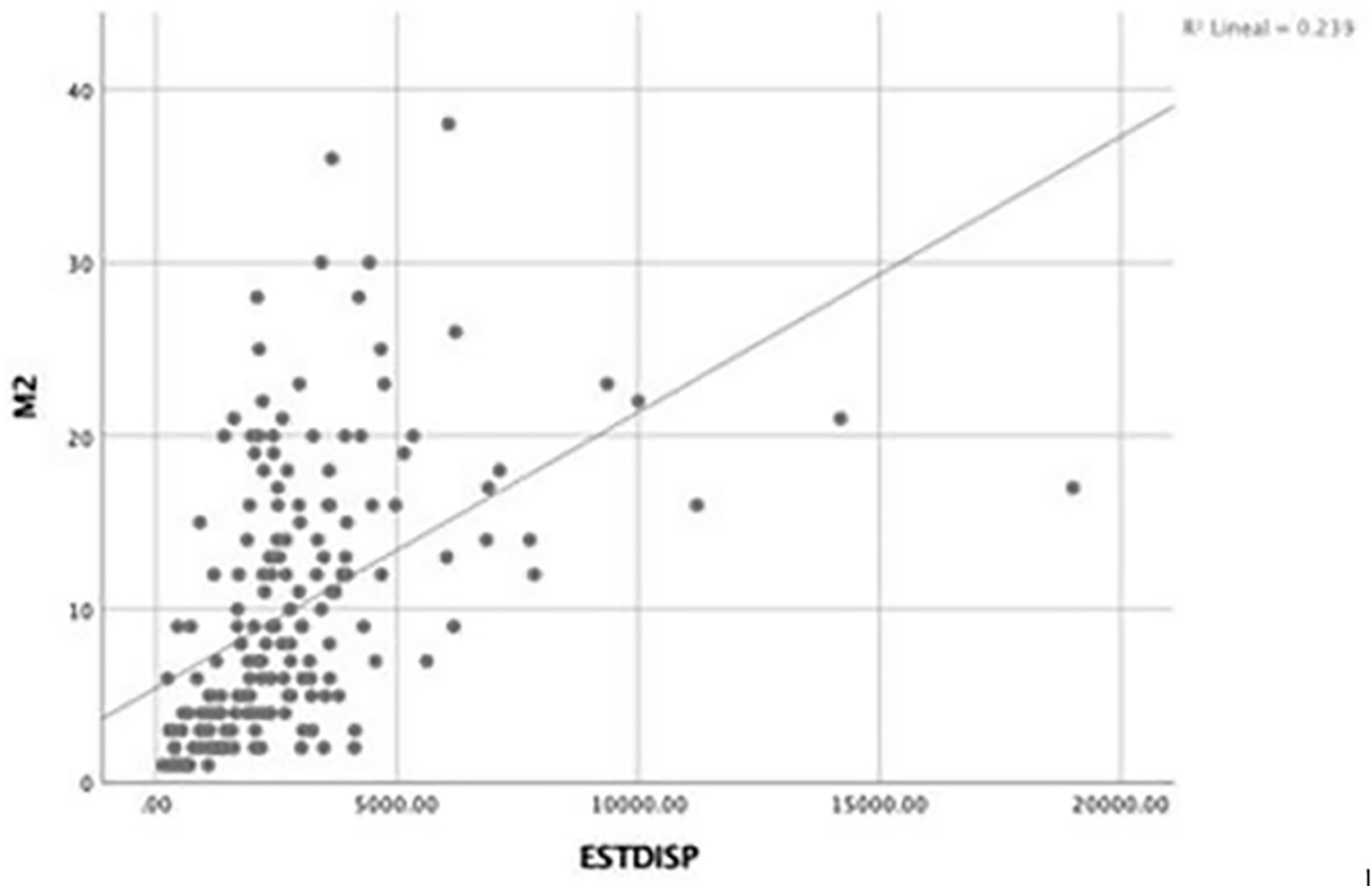

\begin{tabular}{|c|c|c|c|c|}
\hline \multicolumn{5}{|c|}{ Analysis of variance (retrieved oocytes) } \\
\hline Source (model) & gl & Sum ${ }^{2}$ & $\mathrm{~F}$ & $p$ value \\
\hline Regression & 1 & 2554.154 & 55.84 & 0.000 \\
\hline Residual & 178 & 8141.62 & & \\
\hline \multicolumn{5}{|l|}{ Coefficients (retrieved oocytes) } \\
\hline Model term & Estimation & Error tip & $\mathrm{T}$ & $p$ value \\
\hline Constant & 5.447 & 0.779 & 6.988 & 0.000 \\
\hline Estradiol levels on trigger day & .002 & 0.000 & 7.473 & 0.000 \\
\hline
\end{tabular}

Figure 2. Analysis between the number of MII oocytes and E2 levels on the day of trigger.

Table 2. Main findings according to estradiol levels in three study groups with different E2 levels

\begin{tabular}{|c|c|c|c|c|}
\hline Variable & $\begin{array}{c}\text { Group } 1 \\
\left(E_{2} \text { up to } 1000 \mathrm{pg} / \mathrm{ml}\right)\end{array}$ & $\begin{array}{c}\text { Group } 2 \\
\left(E_{2} \text { of } 1001-3000 \mathrm{pg} / \mathrm{ml}\right)\end{array}$ & $\begin{array}{c}\text { Group } 3 \\
\left(E_{2} \text { higher than } 3000 \mathrm{pg} / \mathrm{ml}\right)\end{array}$ & $p$ value \\
\hline Age (years) & $36.7 \pm 5.93(19-46)$ & $35 \pm 6.51(18-56)$ & $37.05 \pm 5.67(18-47)$ & 0.106 \\
\hline Baseline estradiol $(\mathrm{pg} / \mathrm{ml})$ & $49.5 \pm 37.25(5-139)$ & $61.63 \pm 69.58(9-278)$ & $44.95 \pm 22.08(9-104)$ & 0.358 \\
\hline Baseline FSH $(\mathrm{IU} / \mathrm{ml})$ & $5.25 \pm 3.36(0.2-14.7)$ & $9.97 \pm 5.24(1.4-10.9)$ & $8.45 \pm 12(2.7-70.6)$ & 0.362 \\
\hline $\begin{array}{l}\text { Follicles at the end of } \\
\text { stimulation }(n)\end{array}$ & $12.19 \pm 9.27(0-34)$ & $13.35 \pm 9.76(1-48)$ & $9.7 \pm 4.81(1-19)$ & 0.179 \\
\hline Retrieved oocytes (n) & $3.77 \pm 3.32(1-15)$ & $10.65 \pm 7.28(1-33)$ & $16.51 \pm 8.28(2-38)$ & 0.000 \\
\hline Retrieved MII oocytes (n) & $3.1 \pm 3.11(1-15)$ & $9.24 \pm 6.54(1-28)$ & $14.16 \pm 6.54(2-38)$ & 0.000 \\
\hline Oocyte maturity (\%) & 82.2 & 88 & 85 & 0.000 \\
\hline Fertilized $(n)$ & $2.35 \pm 2.65(0-13)$ & $6.48 \pm 5.61(0-23)$ & $10.52 \pm 7.49(0-30)$ & 0.000 \\
\hline Fertilization rate (\%) & 75.8 & 70.12 & 74 & 0.000 \\
\hline
\end{tabular}

Table 3. Main findings according to estradiol levels in three study groups and their different reproductive results

\begin{tabular}{|c|c|c|c|c|c|}
\hline Variable & $\begin{array}{l}\text { Group } 1 \\
\left(E_{2} \begin{array}{l}\text { up to } 1000 \\
\mathrm{pg} / \mathrm{ml})\end{array}\right.\end{array}$ & $\begin{array}{c}\text { Group } 2 \\
\left(E_{2} \text { of } 1001-\right. \\
3000 \mathrm{pg} / \mathrm{ml})\end{array}$ & $\begin{array}{c}\text { Group } 3 \\
\left(E_{2} \text { higher than }\right. \\
\mathbf{3 0 0 0} \mathrm{pg} / \mathrm{mI})\end{array}$ & Kruskal- Wallis H & $p$ value \\
\hline Pregnancy rate $(\%)$ & 32 & 34.8 & 32.8 & 0.102 & 0.950 \\
\hline Miscarriage rate $(\%)$ & 16 & 13.5 & 11.5 & 0.083 & 0.959 \\
\hline Live birth rate (\%) & 22.6 & 11.5 & 21.3 & 0.435 & 0.804 \\
\hline
\end{tabular}


levels were correlated with pregnancy (Blazar et al., 2004) and low $E_{2}$ levels were associated with decreased pregnancy rates (Kondapalli et al., 2012).

Controversy is present in studies, with reports of associations between estradiol to mature oocyte ratios greater than $400 \mathrm{pg} / \mathrm{ml} /$ oocyte in normal responders yielding lower clinical pregnancy (CPR), ongoing pregnancy (OPR) and live birth (LBR) rates than individuals treated in regimens with $<200 \mathrm{pg} / \mathrm{ml} /$ oocyte and 200-300 pg/ml/oocyte (CPR $1 \%$ vs. $16 \%$ and $32 \%$, respectively, $p=0.017$; OPR 0 vs. $15 \%$ and $27 \%$, respectively, $p=0.011$; and LBR 0 vs. $13 \%$ and $26 \%$, respectively, $p=0.018$ ) (Sandoval et al., 2016).

Previous studies reported that the ideal $E_{2}$ range to achieve pregnancy in assisted reproduction cycles is between 1000 to $3148 \mathrm{pg} / \mathrm{mL}$ (Sandoval et al., 2016; Kondapalli et al., 2012). This range is broad and unspecific, and shows that the use of this parameter may not be reliable, like many other hormonal markers that have been used to predict pregnancy rates, such as the AMH (Tal et al., 2015). In our study, the ideal estradiol level to achieve pregnancy was not analyzed, although group 2 had a pregnancy rate $34.8 \%$ higher than the rates seen in groups 1 and $2(p=0.950)$.

It is worth mentioning that low pregnancy rates may be associated with estradiol levels, an essential element in endometrial and placental development. Simón et al. (1995) demonstrated that elevated estradiol levels negatively affected endometrial receptivity and that elevated levels in fresh cycles before transfer correlated with worse implantation outcomes. Farhi et al. (2010) reported that elevated $\mathrm{E}_{2}$ levels during IVF cycles were linked to a higher incidence of adverse pregnancy outcomes.

Future studies should assess whether patients prescribed GnRH analog trigger due to high estradiol levels might suffer from adverse effects other than the risk of ovarian hyperstimulation such as increased miscarriage rates and, paradoxically, lower pregnancy rates, as observed by some authors (Steward et al., 2015).

\section{CONCLUSIONS}

Numerous studies hake looked into estradiol levels on the day of trigger and their impact on ovarian stimulation cycles. However, as mentioned above, few have been conducted to predict the association between estradiol levels, metaphase II oocytes and their correlation with pregnancy rates.

Estradiol is critical in endometrial and placental development; however, excess $\mathrm{E}_{2}$ in the early stages of pregnancy may have adverse effects on placentation. Elevated $E_{2}$ has been shown to adversely affect endometrial receptivity; and high $\mathrm{E}_{2}$ levels in fresh cycles prior to transfer have been correlated with decreased implantation rates. Additionally, elevated $E_{2}$ levels during IVF cycles have been linked to increased incidence of adverse pregnancy outcomes, including preeclampsia and intrauterine growth restriction (Hagerman \& Villee, 1953; Simón et al., 1995; Farhi et al., 2010).

Serum estradiol levels of $308.6 \mathrm{pg} / \mathrm{ml} / \mathrm{oocyte}$ collected on the day of trigger and on the day of egg retrieval during high complexity assisted reproduction cycles with antagonist protocols have been correlated with the number of retrieved oocytes and MII oocytes. Therefore, increases or decreases in one parameter affect its relationship with other parameters regardless of age, although they have not been associated with achieving or predicting pregnancy $(p>0.05)$.

The limitations of this study include its retrospective nature, which makes it impossible to strictly control the factors affecting estradiol levels. Fortunately, in this study all measurements were made in the same laboratory coordinated by the same medical team. The next steps in this scarcely researched topic might include collecting more patient data, conducting more extensive studies to look into each specific situation, and perform randomized and prospective studies.

Studies enrolling specific populations serve as the foundation for epidemiological studies developed to institute personalized patient care based on findings derived from patients with different genetic traits under diverse environmental conditions treated in different corners of the world. The development of individualized treatment protocols that harness the power of predictive tools is one of the goals of future medical practice and patient management.

\section{ACKNOWLEDGMENTS}

The author would like to thank Mr. Héctor Salvador Godoy Morales, MD, for his teachings and for granting access to vast clinical information, and the co-authors for their invaluable support in terms of knowledge and the time they took to collect information from clinical records.

\section{CONFLICT OF INTEREST}

The authors have no conflicts of interest to declare.

\section{Corresponding author:}

Miguel Loyo Guiot

Hospital Angeles Pedregal Assisted Reproduction

Mexico City - Mexico

E-mail:dr.mloyo@hotmail.com

\section{REFERENCES}

Agard J, Glujovsky D, Shamonki MI, Frattarelli J, Bergh PA. Estradiol levels after human chorionic gonadotropin (hCG) administration are not predictive of IVF outcome: analysis of 7,474 initial fresh IVF cycles. Fertil Steril. 2007;88:S125. DOI: $10.1016 /$ j.fertnstert.2007.07.401

Blazar AS, Hogan JW, Frankfurter D, Hackett R, Keefe DL. Serum estradiol positively predicts outcomes in patients undergoing in vitro fertilization. Fertil Steril. 2004;81:1707-9. PMID: 15193504 DOI: $10.1016 / j . f e r t n-$ stert.2003.10.039

Brown JB. Pituitary control of ovarian function--concepts derived from gonadotrophin therapy. Aust $\mathrm{N} \mathrm{Z} \mathrm{J}$ Obstet Gynaecol. 1978;18:46-54. PMID: 278588 DOI: 10.1111/j.1479-828X.1978.tb00011.x

Carmona F, Balasch J, Creus M, Fábregues F, Casamitjana R, Cívico S, Vidal E, Calafell JM, Moreno V, Vanrell JA. Early hormonal markers of pregnancy outcome after in vitro fertilization and embryo transfer. J Assist Reprod Genet. 2003;20:521-6. PMID: 15035553 DOI: 10.1023/B:JARG.0000013654.85531.ac

Farhi J, Ben-Haroush A, Andrawus N, Pinkas H, Sapir O, Fisch B, Ashkenazi J. High serum oestradiol concentrations in IVF cycles increase the risk of pregnancy complications related to abnormal placentation. Reprod Biomed Online. 2010;21:331-7. PMID: 20688571 DOI: 10.1016/j. rbmo.2010.04.022

Hagerman DD, Villee CA. Effects of estradiol on the metabolism of human endometrium in vitro. J Biol Chem. 1953;203:425-31. PMID: 13069526 DOI: 10.1016/S00219258(19)52653-9 
Kondapalli LA, Molinaro TA, Sammel MD, Dokras A. A decrease in serum estradiol levels after human chorionic gonadotrophin administration predicts significantly lower clinical pregnancy and live birth rates in in vitro fertilization cycles. Hum Reprod. 2012;27:2690-7. PMID: 22752608 DOI: $10.1093 /$ humrep/des216

Macklon NS, Stouffer RL, Giudice LC, Fauser BC. The science behind 25 years of ovarian stimulation for in vitro fertilization. Endocr Rev. 2006;27:170-207. PMID: 16434510 DOI: $10.1210 /$ er.2005-0015

Pittaway DE, Wentz AC. Evaluation of the exponential rise of serum estradiol concentrations in human menopausal gonadotropin-induced cycles. Fertil Steril. 1983;40:763-7. PMID: 6418575 DOI: 10.1016/s0015$0282(16) 47476-5$

Sandoval JS, Steward RG, Chen C, Li YJ, Price TM, Muasher SJ. High Peak Estradiol/Mature Oocyte Ratio Predicts Lower Clinical Pregnancy, Ongoing Pregnancy, and Live Birth Rates in GnRH Antagonist Intracytoplasmic Sperm Injection Cycles. J Reprod Med. 2016;61:116. PMID: 26995882
Simón C, Cano F, Valbuena D, Remohí J, Pellicer A. Clinical evidence for a detrimental effect on uterine receptivity of high serum oestradiol concentrations in high and normal responder patients. Hum Reprod. 1995;10:2432-7. PMID: 8530680 DOI: 10.1093/oxfordjournals.humrep.a136313

Speroff L, Fritz MA. Endocrinología ginecológica clínica y esterilidad. Madrid: Wolters Kluwer / Lippincott Williams \& Wilkins; 2007.

Steward RG, Zhang CE, Shah AA, Yeh JS, Chen C, Li YJ, Price TM, Muasher SJ. High Peak Estradiol Predicts Higher Miscarriage and Lower Live Birth Rates in High Responders Triggered with a GnRH Agonist in IVF/ICSI Cycles. J Reprod Med. 2015;60:463-70. PMID: 26775453

Tal R, Tal O, Seifer BJ, Seifer DB. Antimüllerian hormone as predictor of implantation and clinical pregnancy after assisted conception: a systematic review and meta-analysis. Fertil Steril. 2015;103:119-30.e3. PMID: 25450298 DOI: $10.1016 /$ j.fertnstert.2014.09.041

Wu $\mathrm{CH}$, Kuo TC, Wu HH, Yeh GP, Tsai HD. High serum estradiol levels are not detrimental to in vitro fertilization outcome. Taiwan J Obstet Gynecol. 2007;46:54-9. PMID: 17389191 DOI: $10.1016 / \mathrm{S} 1028-4559(08) 60108-4$ 